\title{
Acute tumor lysis syndrome after anti-pd-1 immunotherapy nivolumab for metastatic melanoma.
}

\author{
Daniel Brunnhoelzl ${ }^{1}$ and Jue Wang* \\ ${ }^{1}$ Creighton University School of Medicine at St. Joseph's Hospital and Medical Center, Phoenix, AZ, USA \\ ${ }^{2}$ University of Arizona Cancer Center at Dignity Health St. Joseph's Hospital and Medical Center, Phoenix, AZ, USA
}

Accepted on 27 February, 2017

Keywords: Checkpoint inhibitors; Anti-PD-1 Immunotherapy; Nivolumab; Tumor lysis syndrome (TLS); Metastatic melanoma; Oncological emergency.

\section{Background}

Tumor lysis syndrome (TLS) is an oncological emergency caused by the sudden, rapid death of cancer cells spontaneously or after chemotherapy, commonly in patients with aggressive lymphomas and leukemias, [1-3]. Nivolumab is a fully humanized, monoclonal, immunoglobulin G4 (IgG4) antibody to PD-1 $[4,5]$. To the best of our knowledge, TLS was rarely described in patients with malignant melanoma, and TLS associated checkpoint inhibitors has not been reported in literature.

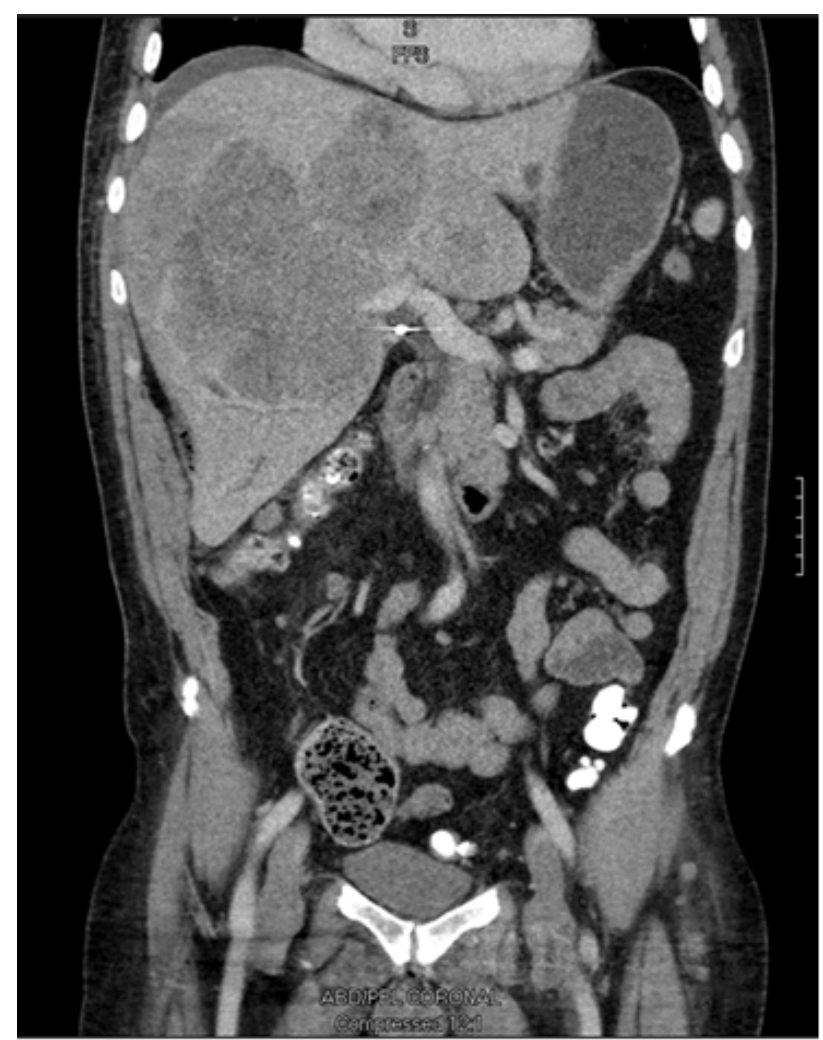

\section{Case presentation}

A 76 -year-old male with history of multifocal melanoma involving maxillary and left nostril (T3NxM0) diagnosed in June 2015, who was treated at outside hospital with resection and adjuvant XRT. One year after initial diagnosis, he presented to our hospital with $20 \mathrm{lb}$ weight loss, fatigue and abdominal bloating. A computerized tomography (CT) scan of the chest abdomen and pelvis with and without IV contrast revealed widely metastatic disease involving lung, liver, kidneys, omentum, adrenal, bones (Figure 1).

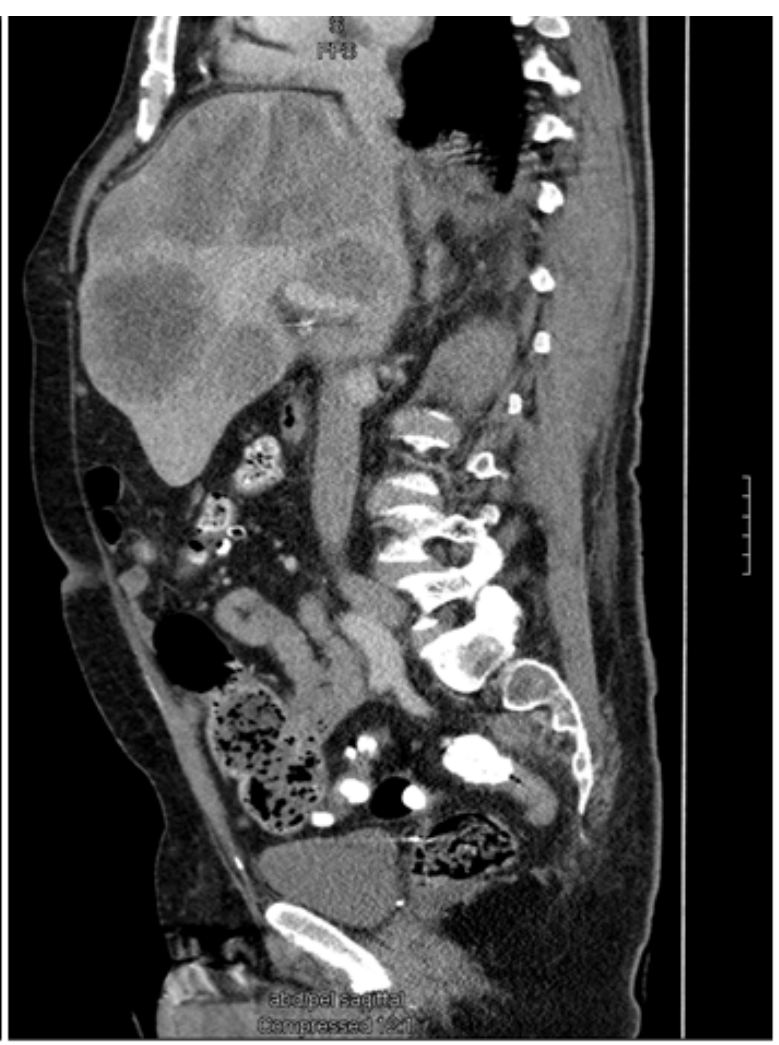

Figure 1: A computerized tomography (CT) scan of the chest abdomen and pelvis with and without IV contrast showed diffused metastatic disease.

A biopsy of liver lesion was consistent with metastatic melanoma (No mutation was found in C-kit and BRAF). After a thorough oncological evaluation, the decision was made to treat recurrence disease with Nivolumab. Five days after the third dose of treatment, he was admitted to hospital for altered mental status. His laboratory results were notable for hyperkalemia with potassium $6.7 \mathrm{mmol} / \mathrm{L}$ (Normal Range 3.6-5.0), hyperphosphatemia $7.1 \mathrm{mg} / \mathrm{dL}$ (NR 2.4-4.7), calcium $7.3 \mathrm{mg} / \mathrm{dL}$ (NR 8.5-10.3), sodium $136 \mathrm{mmol} / \mathrm{L}$ (NR 135-145), chloride $102 \mathrm{mmol} / \mathrm{L}$ (NR 100-110), carbon dioxide $16 \mathrm{mmol} / \mathrm{L}$ (NR 19-27), BUN $110 \mathrm{mg} / \mathrm{dL}$ (NR 8-25), creatinine $3.9 \mathrm{mg} / \mathrm{dL}$ (NR 0.57-1.11), anion gap $16 \mathrm{mmol} / \mathrm{L}(\mathrm{NR} 7-15)$, and uric acid 
Citation: Brunnhoelzl D, Wang J. Acute tumor lysis syndrome after anti-pd-1 immunotherapy nivolumab for metastatic melanoma.. J Mol Oncol Res. 2017;1(1):5-6.

$15.9 \mathrm{mg} / \mathrm{dL}$ (NR 2.6-6.3). The patient was treated with aggressive intravenous (IV) rehydration and nephrology was consulted. Unfortunately, his condition deteriorated rapidly. The patient's family declined hemodialysis, and decided to take him home with hospice care (Table 1).

Table 1: Blood chemistry values in a patient with acute tumor lysis syndrome.

\begin{tabular}{|c|c|c|}
\hline & 3 weeks before PTA & Admission \\
\hline BUN (mg/dL) & 22 & 110 \\
\hline $\begin{array}{l}\text { Serum } \\
\text { (mg/dL) }\end{array}$ & 0.85 & 3.9 \\
\hline $\begin{array}{l}\text { Serum } \\
\text { (mg/dL) }\end{array}$ & 2.4 & 7.8 \\
\hline $\begin{array}{l}\text { Serum } \\
(\mathrm{mEq} / \mathrm{L})\end{array}$ & 4.5 & 7.1 \\
\hline Serum sodium (mEq/L) & 136 & 132 \\
\hline Serum calcium (mg/dL) & 9.5 & 7.3 \\
\hline Uric acid (mg/dL) & 3.8 & 15.9 \\
\hline
\end{tabular}

\section{Conclusions}

Checkpoint inhibitors have revolutionized the treatment of patients with metastatic melanoma, offering improved responses and significant survival benefit. Understanding the potential complications of these novel therapies is critical in optimal clinical management of patients with metastatic melanoma. Our case demonstrates that administration of antiPD-1 immunotherapy nivolumab may induce acute tumor lysis syndrome. Considering those immunotherapies are increasingly used in advanced cancer, oncologists should be alert to this potential complication. Frequent laboratory monitoring, appropriate prophylaxis and prompt initiation of appropriate therapy are advised when treating elderly patients at high risk of tumor lysis syndrome.

\section{References}

1. Howard SC, Jones DP, Pui CH. The tumor lysis syndrome. N Engl J Med. 2011; 364(19): 1844-54.

2. Mott FE, Esana A, Chakmakjian C, et al. Tumor lysis syndrome in solid tumors. Support Cancer Ther. 2005; 2: 188-91.

3. Coiffier B, Altman A, Ching-Hon P, et al. Guidelines for the management of pediatric and adult tumor lysis syndrome: an evidence-based review. J Clin Oncol. 2008; 26(16): 2767-78.

4. Luke JJ, Ott PA. PD-1 pathway inhibitors: the next generation of immunotherapy for advanced melanoma. Oncotarget. 2015; 6(6): 3479-92.

5. Robert C, Long GV, Brady B, et al. Nivolumab in previously untreated melanoma without BRAF mutation. $\mathrm{N}$ Engl J Med. 2015; 372: 320-30.

\section{*Correspondence to}

Jue Wang, MD

University of Arizona Cancer Center at Dignity Health St. Joseph's, Phoenix, AZ

$625 \mathrm{~N}$ 6th Street,

Phoenix, AZ 85004

Tel: +1 602-406-8222

E-mail: jue.wang@dignityhealth.org 